\title{
Acute pancreatitis and diabetic ketoacidosis in non-diabetic psychotic patient.
}

Ibtissem Oueslati, Emna Elfeleh, Karima Khiari, Néjib Ben Abdallah

Department of Endocrinology, Charles Nicolle hospital. Tunis, Tunisia

\section{INTRODUCTION :}

Simultaneous occurrence of acute pancreatitis and diabetic ketoacidosis represents a very rare complication of antipsychotic medications.

Herein we report the case of acute pancreatitis and diabetic ketoacidosis in a nondiabetic patient while on treatment with haloperidol, chlorpromazine and carbamazepine.

\section{CASE REPORT:}

Mr. Z A is a 50 year old non diabetic man who was diagnosed with psychotic disorder and treated with haloperidol, chlorpromazine and carbamazepine.

There were no past or present history of alcohol use and no history of gallstones or trauma.

In September 2015, the patient presented with sudden onset weakness and vomiting. On examination, he had a body weight of $110 \mathrm{~kg}$, a body mass index of $40.4 \mathrm{~kg} / \mathrm{m}^{2}$ and he was tachycardic and tachypnoeic.

Abdominal examination revealed tenderness in the epigastrium.

Capillary glycemia was $3.56 \mathrm{~g} / \mathrm{l}$ with glycosuria=+++ and acetonuria=+++.

On laboratory tests, he had a glycemia of $24 \mathrm{mmol} / \mathrm{l}$, a bicarbonate level $<3 \mathrm{mmol} / \mathrm{l}$ and an acute inflammation. Lipase was elevated at $1024 \mathrm{U} / \mathrm{l}(\mathrm{nr}=7-60)$. Calcemia, lipid and liver function tests were normal.

Abdominal CT-scan showed acute pancreatitis features.

Patient was managed with insulin and intravenous fluids with a good recovery.

\section{CONCLUSION :}

In our patient, features supporting acute pancreatitis secondary to antipsychotic drugs include the absence of alcohol intake, trauma or gallstones and normal lipids and calcemia. The possibility of developing acute pancreatitis and diabetic ketoacidosis while treated with antipsychotic agents impose periodic metabolic screening 